\title{
First experiments for the diagnosis and thermophysical sampling using impulse IR thermography from Unmanned Aerial Vehicle (UAV)
}

\author{
by L. E. Mavromatidis ${ }^{*}$ J.-L. Dauvergne ${ }^{* *}$, R. Saleri*, J.-C. Batsale ${ }^{* \star \star}$
}

\author{
* MAP-Aria Laboratory, UMR CNRS-MCC 3495, ENSAL, 3 rue Maurice Audin, BP 170, F-69512 Vaulx-en-Velin Cedex, \\ France, lazarosmavr@hotmail.com; renato.saleri@lyon.archi.fr \\ ** Epsilon Fahrenheit, Esplanade des Arts et Métiers, F-33400 Talence, France, jldauvergne@epsilon-alcen.com \\ *** Univ. Bordeaux, I2M, UMR 5295, F-33400 Talence, France, jc.batsale@i2m.u-bordeaux1.fr
}

\begin{abstract}
Infrared (IR) thermography is a control method widely used for building diagnosis to investigate structural blemishes and thermal heat losses. Usually, collecting the thermal heat flux naturally emitted by a studied surface via an IR camera, we obtain critical information regarding its structure through passive infrared thermography. The thermogram can then reveal an abnormal variation of the heat flux and highlight a defection. However, on building applications in the case of Non Destructive Testing and Energy Consumption Investigation, spare control often only emits no significant heat due to the high thermal inertia materials that are often used. To overcome such problem, active impulse infrared thermography is an interesting alternative method, since we can access to information not provided spontaneously by analyzing the passive thermal emission of the surface. Hence, in this work, the front face flash method is proposed in order to analyse the subsurface thermal properties. Furthermore, it is proposed to couple impulse IR thermography with a stable Unmanned Aircraft Systems (UAV) to record and analyse the transient temperature response during convenient time duration. Preliminary results and several practical problems for the implementation of such experimental device are given and discussed here.
\end{abstract}

\section{Introduction}

According to international normative standards the enhanced amount of old civil infrastructure and residential buildings has become an important issue regarding their energy consumption, their ageing process and hence life cycle management. Conventional means for monitoring the condition of those buildings could not provide the information needed to perform detailed simulation in order to obtain accurate results regarding their thermal performance. Classic UAV mainly provide integral information about cracking condition and possibly detachment of the covering layers of concrete or stone based structures, however, impulse IR thermography from unmanned aerial vehicle could provide more precise information regarding structural degradation on exterior layers and thermophysical sampling. Furthermore, conducting impulse IR investigation we potentially could be able to estimate with accepted accuracy thermophysical properties of building's constructive material and hence we could be able to estimate the influence of ageing in thermal performance issues. Effort required in providing this information can become laborious when considering structures of a high altitude since significant lifting equipment is required for such inspection. To circumvent this effort the use of UAV and at small scales of micro aerial vehicles (MAV) equipped with high resolution thermal imaging camera to capture the required data is used in the framework of this paper.

The main purpose of this work is to introduce a simple experimental protocol regarding the realization of simple flash methods combined with UAV active IR thermography systems on large surfaces (for instance for buildings' evaluations). This first approach has as main objective to investigate and evaluate the technical performances of such a system. The main principle and aim of the experiment is on the one hand to employ a UAV equipped with an IR camera (Figure 1); on the other hand to use a flash lamp that could be implemented independently from the UAV on a stable position or on a second UAV. The first experimental approaches and the technical problems to implement such devices are presented here and compared to classic laboratory experiments.

In the framework of this study, a rotary wing octocopter micro air vehicle (MAV) system (Push-Pull Octocopter DS8X-N3) equipped with a high resolution thermal imaging camera (Flir A320), has been used in laboratory conditions to scan surfaces for defection diagnosis and thermophysical sampling purposes. The MAV has been equipped with a microcontroller based flight control system and different sensors for manual navigation and flight stabilization. Thermograms have been taken at a high speed and frequency, following a real time computer stored procedure while the MAV was up in the air. The IR camera was continuously connected to a PC monitor and provided real-time radiometric thermal images that accurately revealed heat patterns and thermal anomalies. According to the flight protocol the MAV system flight from 2 up to $8 \mathrm{~m}$ distance from the samples while keeping the same altitude throughout the entire experimental procedure. Taken IR images have then been analyzed together to obtain a full 2D image having as main aim to observe damages and cracking in the millimeter range. Furthermore, these pictures have been compared to pictures obtained from stable positioning of the active IR camera system for the same samples and under the same conditions. In a follow-on step an image processing software developed on the LabVIEW environment has been employed while software that allows cracking patterns to be specifically filtered out for further pattern stabilization to erase noising due to MAV's instabilities has been used in a future step.

Thus, in the present paper we present a first experiment that consists in irradiating uniformly the surface of a 
selected sample to compare the average temperature decrease of a given surface of interest obtained through a classic laboratory experiment to the response that resulted from coupled impulse IR-UAV experimental sequences. The experiments showed that if a large area of interest can be defined in detail, it is possible to obtain reliable results without precautions regarding the stability of the UAV. Furthermore, to evaluate the accuracy of the coupled IR-UAV system a second experiment has been realized where we considered a localized hot spot to estimate the influence of the stability of the UAV. The methods used here were transient infrared thermography, while both flash heating and step heating thermography was studied.

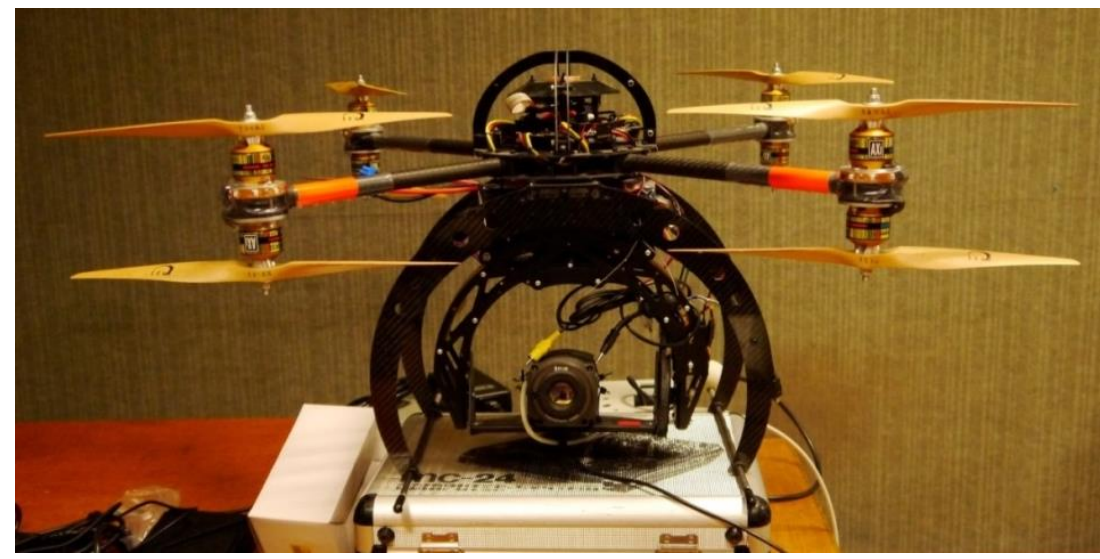

Fig 1. UAV (Push-Pull Octocopter DS8X-N3) equipped with an IR camera (Flir A320G)

\section{Non destructive evaluation methods}

\subsection{Choice of active thermography and front flash method}

Non Destructive Evaluation Methods (NDE) play a growing role in all aspects of materials production and their applications. By definition, the NDE uses different techniques of non-intrusive measures to verify the integrity of a structure, component or material without destroying it. The thermal methods are one of them. The main principle consists in applying a transient non-contact radiative heating at the surface of the studied medium (generally a heat pulse or a periodic heating) and by analysing the surface temperature mapping generally obtained with an infrared camera. Such methods are very different than the static observation of the infrared surface emission (passive thermography) because they avoid to consider the influence of the reflections (assumed to be stationnary) of the environment around the considered surface. The transient signal coming from the IR camera can then be conveniently considered as proportional to the transient temperature field without knowing the absolute temperature level.

In the case of UAV experiments, for practical reasons, only a short pulse heating can be implemented and then only the front face flash method will be considered.

Two ways of heating can be implemented: a spatially uniform heating allowing to estimate only in-depth properties or a localized spot heating allowing to estimate simultaneously in depth and in-plane thermal diffusivities.

The difficulties of the experiments will give here a noisy and biased signal which will induce difficulties for the processing and parameters estimation.

\subsection{Several approaches for the signal processing related to the heating mode}

\section{-case of a front face uniform heating.}

By anticipating the very noisy signal coming from such experiments it was decided to analyse only the averaged temperature variation of a whole surface without an accurate knowledge of the thermal model related to the signal. In a previous work, it isshown (Mourand and Batsale, 1998) that a temperature response on a front face: $T(Z=0, t)$ can be expressed by a function only depending on the global amplitude of an averaged observed signal $f(t)$ and by the logarithmic time derivative $t \frac{f}{t} \mid$ of this function. For instance, the thermal conductivity variation of a plate of thickness $L$, referred to a nominative thermal conductivity ${ }_{0}$, and a volumetric heat capacity $C$, with a global heat energy $Q$, can be expressed as: 
$T(z=0, t) \approx \frac{Q}{c L} f\left({ }_{0} t / c L^{2}\right)+\left.\frac{Q}{c L} \frac{-t}{{ }_{0}} \frac{f}{t}\right|_{\left(0_{0} t / c L^{2}\right)}$

The sensitivity functions $f(t)$ and $t \frac{f}{t} \mid$ can then be used for the implementation of a linear mean square method. Such methods are convenient in order to reduce the signal noise influence. It is only necessary to dispose of a quite repetitive signal on a logarithmic time scale.

-case of a localised front face heating

In the case of localised heating, we must consider not only the in-depth but also the in-plane heat diffusion. One example of temperature evolution from a punctual heat pulse can be expressed as:

$$
T(x, y, z=0, t)=\frac{Q}{c} \frac{\exp \left(\left(x \quad x_{0}\right)^{2} /\left(4 a_{x} t\right)\right)}{\sqrt{a_{x} t}} \frac{\exp \left(\left(y \quad y_{0}\right)^{2} /\left(4 a_{y} t\right)\right)}{\sqrt{a_{y} t}} \frac{1}{\sqrt{a_{z} t}}
$$

with: $\quad a_{x}, a_{y}, a_{z}:$ Thermal diffusivities versus $x, y, z$ direction $\left(m^{2} s^{-1}\right)$

$$
\begin{aligned}
& Q \text { : Energy of the pulse point source }(\mathcal{J}) \\
& \rho c: \text { Volumetric heat capacity }\left(\mathrm{Jm}^{-3} K^{-1}\right)
\end{aligned}
$$

Unfortunately, in the case of UAV experiments, it is not only very difficult to master the position of the spot, but also the scale of the in-plane coordinates system $(O, x, y)$ which is correlated to the lack of stability of the aerial vehicle. Such methods as those developed by Bamford et al (2006) could not be applied, even if the position of the spot is simultaneously estimated as the thermal diffusivity.

\subsection{The use of UAV on building and urban analysis}

The originality of this work is mainly based on the device employed to perform the front face active IR analysis. Indeed, in our case the infrared camera is attached to a UAV vehicle. This choice offers as main advantage the opportunity to reach inaccessible areas of a structure with a standard installation and perform impulse IR investigation. Nowadays UAV devices are used for many applications such as: assessment of canopy height in discontinuous crop canopy using digital surface models obtained from very high resolution imagery acquired with unmanned aerial vehicles (Zarko-Tejada et al, 2014), urban traffic analysis (Salvo et al, 2014), archaeological aerial thermography (Cassana et al, 2014), scene reconstruction techniques in computer vision field to large scene reconstruction from UAV images (Guo et al, 2013). Furthermore, statistical embedding of structural pattern recognition systems into the autonomous navigation of UAV has been developed for the recognition of visual landmarks such as bridges in aerial views having as a result an optimised navigation (Michaelsen et al, 2014). Moreover, UAV systems are becoming attractive for many surveying applications in civil engineering as data acquisition platforms and as measurement instruments, to rapidly and autonomously acquire mobile three-dimensional (3D) mapping data (Siebert et al, 2014; Remondino et al, 2011; Hudzietz et al, 2011).

Even if the use of UAV devices coupled to IR thermographs seems to be an attractive option there are few studies that focus on the potential of such a system. Calderón et al (2013) explored the use of high-resolution thermal imagery, chlorophyll fluorescence, structural and physiological indices (xanthophyll, chlorophyll $a+b$, carotenoids and blue/green/red B/G/R indices) calculated from multispectral and hyperspectral imagery as early indicators of water stress caused by VW infection and severity. They based their study on the analysis of time series of airborne thermal, multispectral and hyperspectral imagery during three consecutive years and related to VW severity at the time of the flights. The most interesting issue was that concurrently to the classic airborne campaigns, field measurements conducted at leaf and tree-crown levels showed a significant increase in crown temperature (Tc) minus air temperature (Ta) and a decrease in leaf stomatal conductance (G) across VW severity levels, identifying VW-infected trees at early stages of the disease. This study showed that alternative IR techniques coupled to UAV devices could reveal a very interesting potential because of the ability of the UAV to fly close to the investigated area. Furthermore, the most important issue of the above-mentioned study is resumed in the fact that the UAV didn't add noise in the problem due to UAV instabilities and so experimental data provided all the necessary elements to the study. 
In our case, this kind of coupling potential strengths due to the association of active impulse thermography with a mobile device motivated us to perform a detailed data analysis in order to investigate if such a system could be operational. In general, the use of UAV on building applications is limited. Usually, only passive infrared aerial images for building diagnosis on urban scale are produced during the winter, to highlight the defects of roofing insulation with the use of airplanes. They help owners, social housing, companies and communities to prioritize investments regarding energy savings (loft insulation or terrace insulation first, because it is often the most profitable investment in terms of saving energy to achieve the objectives of the Kyoto Protocol).

A UAV or airplane equipped with a thermal infrared sensor can fly over the city to the same usual altitude (500 to 1000 meters depending on the circumstances and the desired accuracy). To reduce the cost of such operation, several French companies now offer a service of drone aerial passive thermography that allows to quickly performing a complete energy consumption diagnosis of a building based on aerial thermography investigation of the roof and the facades. The final heat map is a representation in the register right buildings, luminance recorded in infrared thermography. It also allows stimulating local economic social fabric (improvements of the habitat) and refining the knowledge on the energy consumption of the city's infrastructure (schools, sports halls, administrative and technical buildings, homes, businesses, etc)

In building scale, since portable ground thermographs currently employed for passive IR investigations suffer from the opposite problem of aerial IR configurations, aerial passive thermography has been developed to overpass the lack of perspective that a thermal reflection of the operator may create, but also the difficulty to visualize the roof or other surfaces located on high altitudes. In a general research framework, similarly to Calderón et al (2013) our main purpose here is to conduct an original study that focus on the coupling of UAV with active IR configurations for building applications and explore the feasibility of such a system.

\section{Experimental results and discussion}

\subsection{Experimental setup}

As detailed above, the preliminary experimental device consists of an infrared camera, a computer, a flash source (needed for sample's excitation) and a sample (see Figures 2 and 3). The camera used in these experimental sequences (type A320G, manufactured by Flir) allows data acquisition at $60 \mathrm{~Hz}$ and has the advantage of having relatively small dimensions and mass to facilitate subsequent coupling to the drone and limit its impact during the flight in order to reduce the stabilisation problems. In the framework of initial tests, the camera is mounted on a conventional support and is connected to a PC equipped with software for the acquisition and processing of data. The excitation source (flash lamp XENOLUX 1500) was placed in front of the sample. The sample of this study is a composite plate (2.3mm thickness, $41 \times 30 \mathrm{~cm}^{2}$ area) which has the following advantages: its thermal properties are known (diffusivity equal to $4.5 \mathrm{E}-7 \mathrm{~m}^{2} / \mathrm{s}$ ), it includes a plurality of resistive defects (delamination-type) and is already calibrated to allow for face front impulse IR testing. During testing, the sample is fixed and observed using the infrared camera located at different distances therefrom. After the thermal excitation of the composite sample on one of its faces, its temperature evolution is measured by the infrared camera.

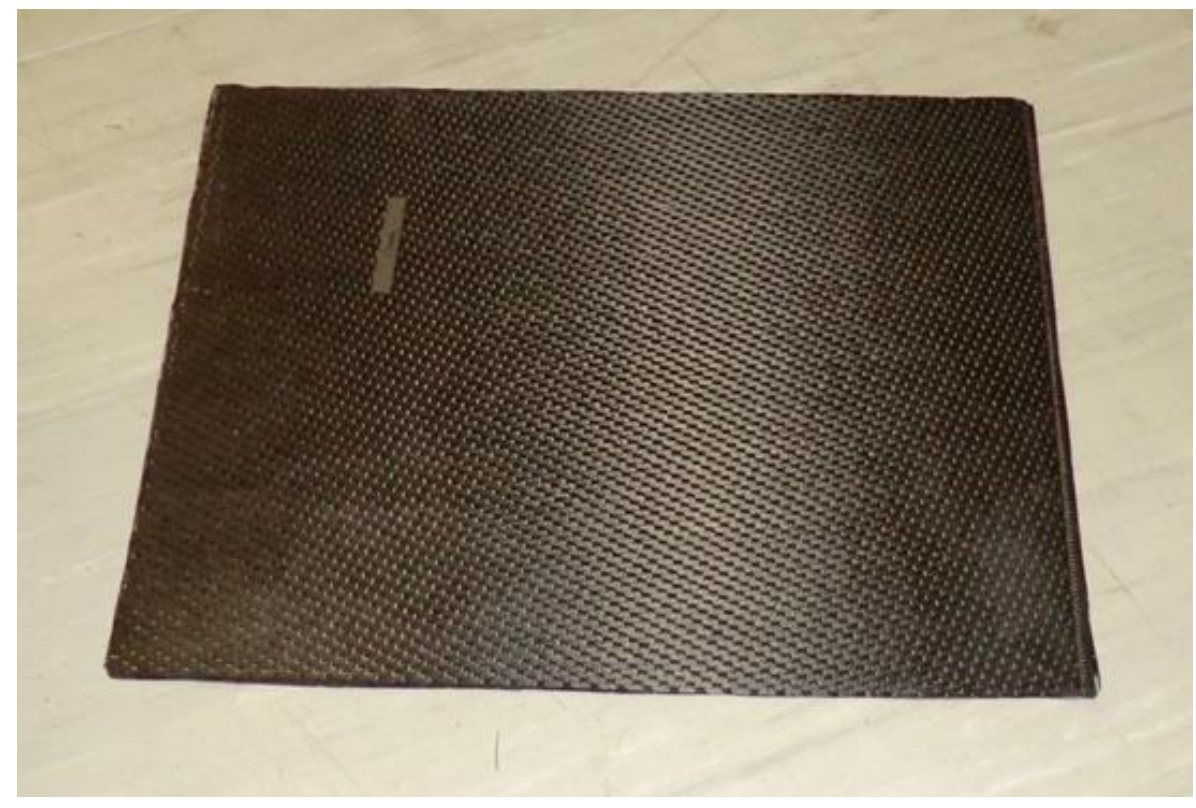

Fig 2. Comparison of front face temperature responses to flash heating in the case of a large uniform irradiation with a fixed camera and a stabilized camera with UAV at two distances $(2 \mathrm{~m}$ and $6 \mathrm{~m}$ ). 

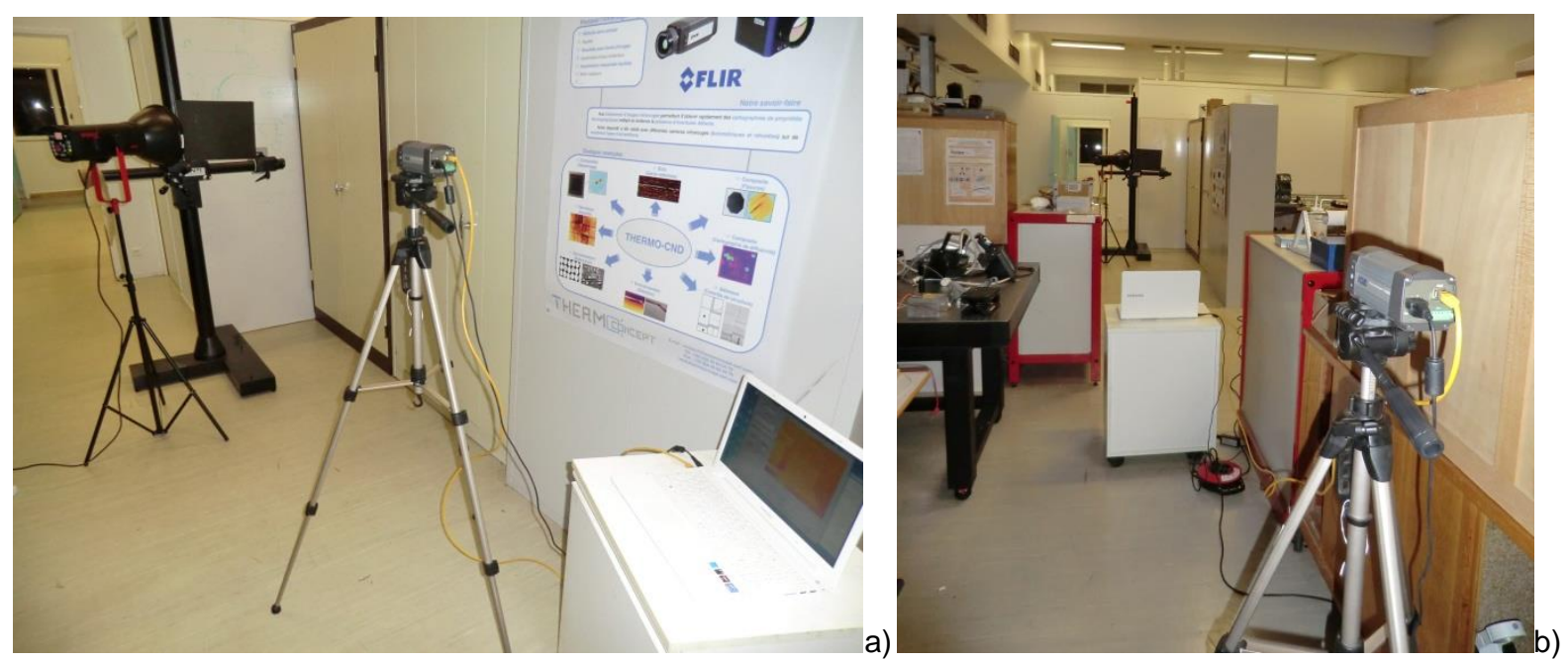

Fig 3. Preliminary experimental setup (front face impulse IR testing). From left to right: camera located at (a) $2 \mathrm{~m}$ and (b) $8 \mathrm{~m}$ distance from the sample.

After taking into account the preliminary results obtained using a fixed IR camera, we repeated the same tests coupling a UAV (Push-Pull Octocopter DS8X-N3) with the same IR camera (Flir A320G) in order to realize the front face impulse IR technique. The technique of exploiting thermograms obtained in the front face of a surface subjected to a flash drive, has two advantages: the first, already mentioned, is that the back side of a given area is not always accessible to the operator, the second is that the tests are shorter than rear face impulse thermography. This helped us to limit the impact of the UAV's instability during the experimental sequences. In this study, the UAV is controlled manually by an operator. On the other hand, the camera having neither battery nor wireless connection, is permanently connected to the grounded with cables (Figure 4a). The combination of these constraints, as we will see later, is an important source of noises during the experimental sequences (erratic stabilization, altitude loss during the test, change of the distance sample-camera, etc.). The sample described in the previous paragraph is again fixed to a support and an identical flash lamp is placed so as to urge its front face (Figure 4b).
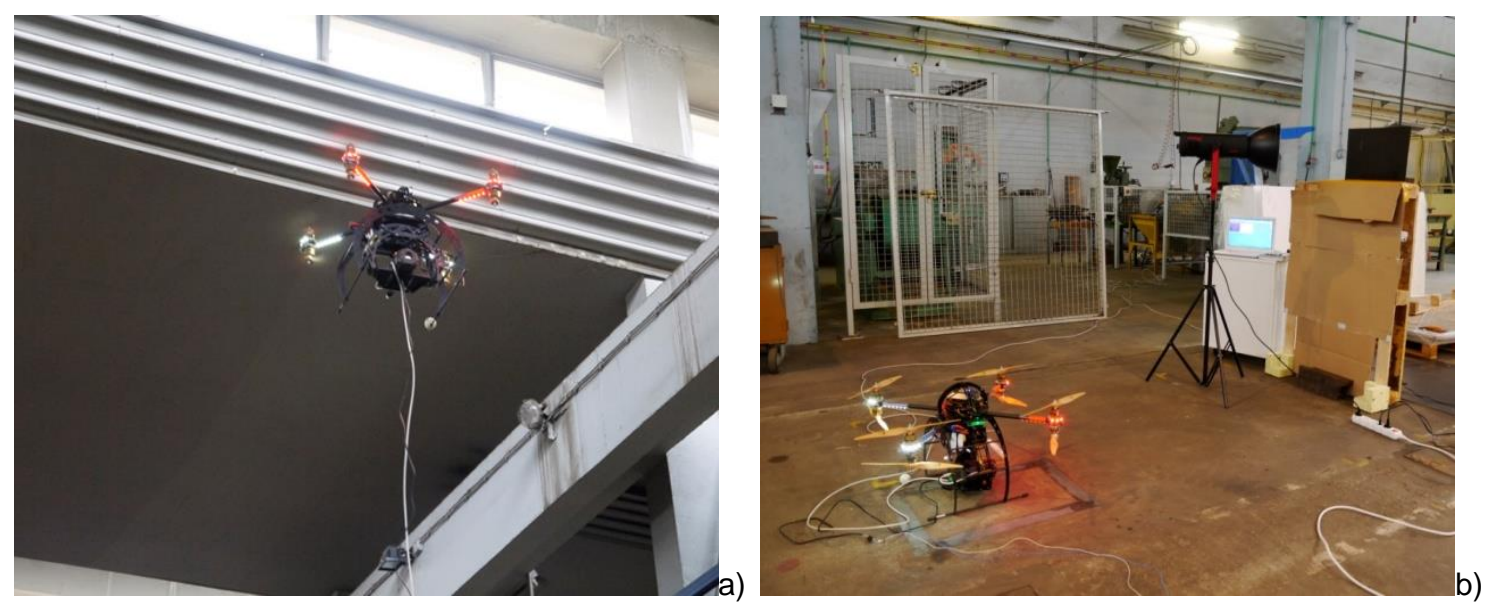

Fig 4. a) Extension cables RJ45and power supply cables; b) full experimental setup for impulse front face IR thermography coupled with a drone.

\subsection{Front face uniform flash confrontation}

As we mentioned in the previous subchapter, the in-flight tests were conducted, on different distances between the camera and the sample. The results are obtained at different sample-camera distances (approximately 2, 6 and $8 \mathrm{~m}$ ). All experiments are implemented with a fixed large flash lamp, a fixed camera and a flying camera installed on the UAV. In order to avoid the stability problems of the UAV, a thermally uniform large area of interest is delimited and averaged to record only one averaged temperature transient evaluation. The temperature responses on fixed and flying device are plotted on figure 5 and compared. 


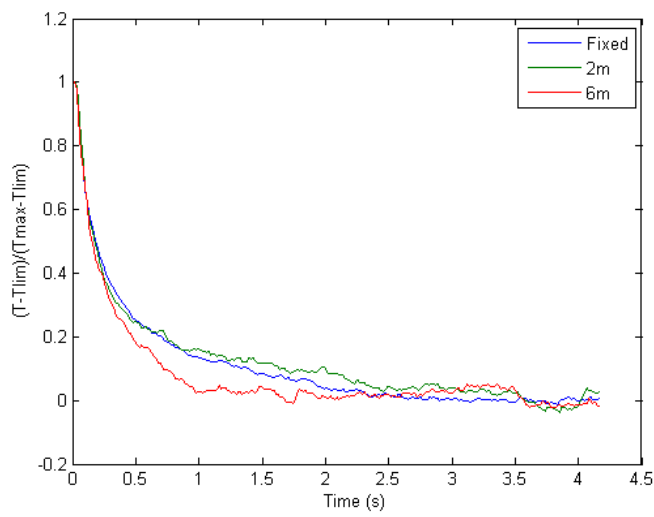

a)

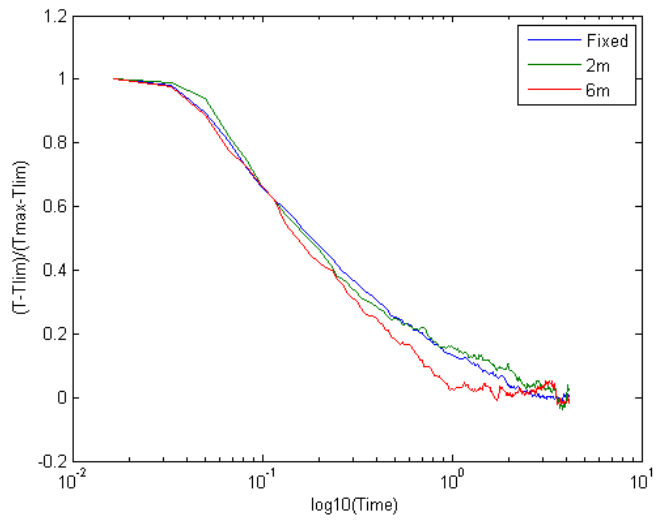

b)

Fig 5. a) Comparison of front face temperature responses to flash heating in the case of a large uniform irradiation with a fixed camera and a stabilized camera with UAV at two distances $(2 \mathrm{~m}$ and $6 \mathrm{~m}$ ) and b)plot of the results in logarithmic coordinates.

The test performed at 8 meters distance highlights the difficulty of this study, since drone's stabilization during the experiment revealed to be a crucial point. Power and communication cables with the computer, as well as manual control, generate damaging to the quality of measurements by adding noise due to the pitch cables. The following images (Figure 6) illustrate its vertical and horizontal variations during the test (taken at $0,2.5$ and 5 seconds during the test from $8 \mathrm{~m}$ distance). These first observations will highlight two axes for improvement of the experimental protocol. Firstly we should fully automate the UAV -by employing a GPS pilot for example-, and remove the connecting to the ground cables (for power and communication purposes) through the use of wireless communication while choosing a battery IR camera. Regarding the reproducibility of the results, we observe on figure 5 that despite the strong pitching, it is possible to obtain comparable thermal normalized responses from distances up to $6 \mathrm{~m}$.
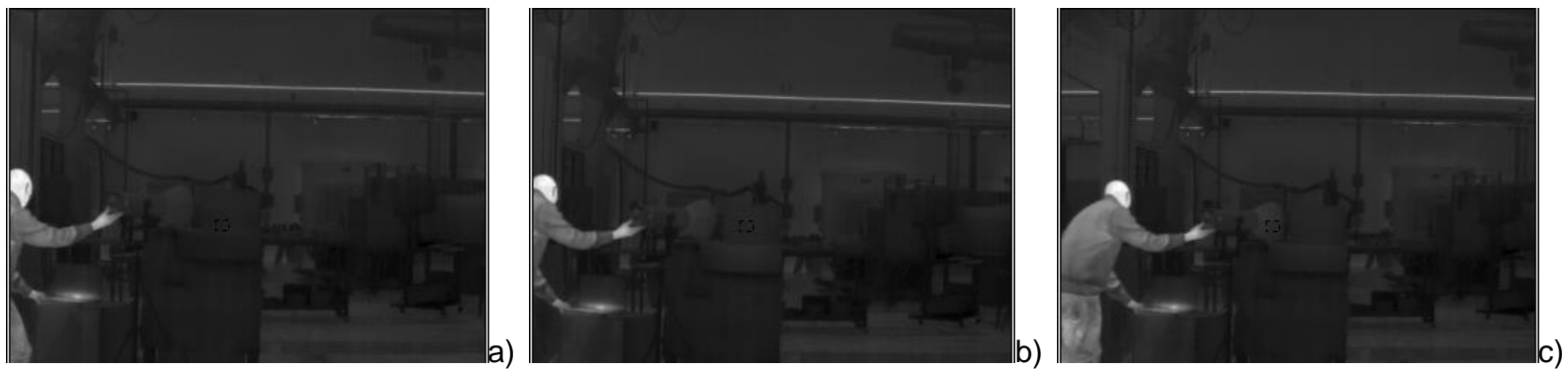

Fig 6. Thermal scene and zone of interest. Aerial views taken from $8 \mathrm{~m}$ distances after: a) 0 sec, b) 2.5 sec, c) 5 sec.

On the other hand, the superposition of these experimental thermograms with those obtained previously from a fixed IR camera (black in Figure 5), shows a satisfactory fit. The results have also been plotted versus a time-logarithmic coordinates and seems to be quite superposed. This confirms that an analysis from expression (1) can be implemented in order to differentiate materials showing significant variations of the thermal properties.

However, even if these results are encouraging, we should denote the purely qualitative nature of such an analysis. In the current state of both experimental device and methods of processing, it is not possible to detect structural defects or to characterize them in detail. Nevertheless, we can imagine other ways of improving these tests. It would be interesting, to perform the same experiments with a stabilized UAV, and develop a system that detects automatically the thermal scene; for example, some clearly identified fixed points during the test will be the reference value to correct afterwards the recorded images. A modification of the excitation source could also provide additional information facilitating the detection of defects. Tests with excitation sources closer to a given niche are thus conceivable. Regarding the same excitation source, its mounting on the UAV in the same axis as the infrared camera, would limit the impact of the inhomogeneity of illumination of the thermal scene.

\subsection{Localised hot spot (test of the stability of the UAV)}

One other point of interest is to be able to examine the case of a hot spot. When the camera is fixed, the hot spot can be analysed in order to estimate the in-plane thermal diffusivity (see Philippi et al, 1995 and Bamford et al, 2006). Such estimation will be very difficult when the camera is flying because the sample landmark is randomly changing. In figure 7, the hot spot at two different times illustrates this problem. Instead of thermophysical properties, the apparent relative velocity of the object in the landmark of the UAV can be estimated with methods for instance developed by Bamford et al., 2006. 

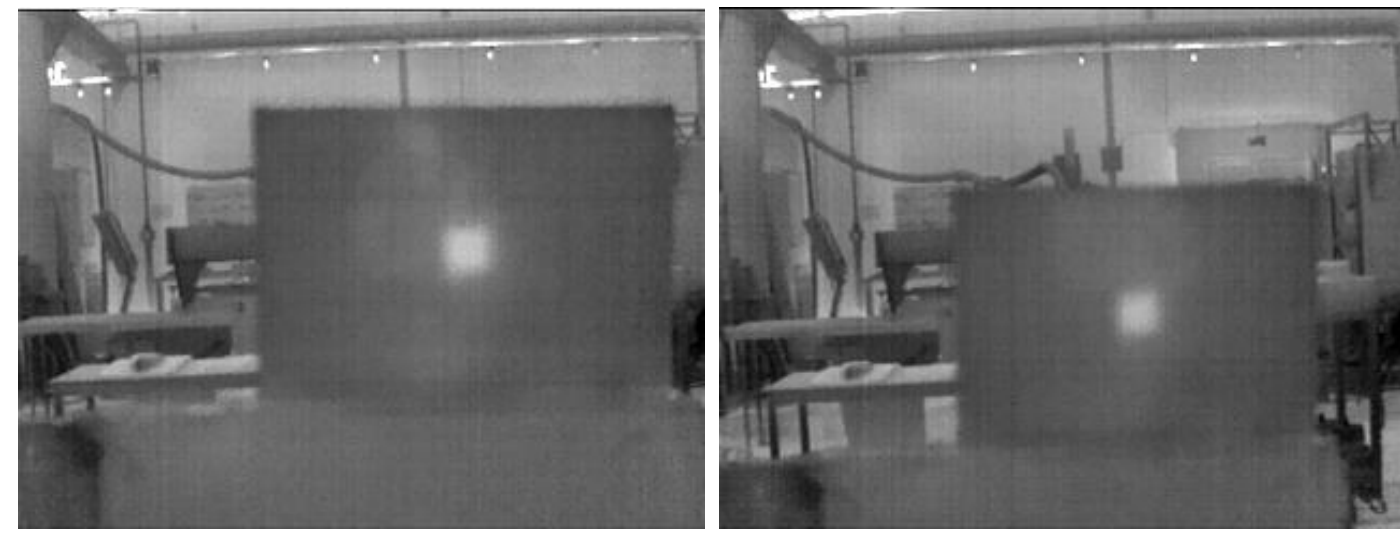

Fig 7. a) Hot spot at $t$ and b) Hot spot at $t+1 \mathrm{~s}$

\section{Conclusions and Perspectives}

This paper presents a variation of the front impulse thermography method while the thermograms are obtained here from an UAV. The present approach has the advantage of thermal exploration of not easily accessible large areas, achieved in cases that are generally out of reach for standard devices. In this exploratory study, it was highlighted the feasibility of obtaining reproducible characteristic thermograms of the impulse thermography pattern. Defects have also been detected in the spatial resolution of the camera. Different areas for improvement can now be considered. Firstly, longer duration tests would be desirable in order to observe the asymptotic behaviour (stationary signal) for a long time and thus allow an accurate estimation of thermal property. On the other hand, it has been shown that in order to obtain mapping properties and defects, it would be necessary to perform a pixel by pixel treatment in addition to the classic study of the average signals. This is particularly difficult at present due to UAV's stabilisation problems. The possibility to conduct UAV's flight automation through GPS pilot is an attractive perspective. It is noteworthy that the removal of connecting cables that link the UAV to the ground (power and connection to PC) by using a wireless communication and choosing a power independent camera could be the first improvement to the experimental setup presented here. Failing to achieve this stabilization, we should develop a system for thermal scene based monitoring, for example, choose some clearly identified during testing fixed points, correct and post-process the recorded images. On the other hand, a modification of the excitation source may also provide additional information to facilitate the detection of defects: experimental sequences with closest excitations should thus been tested. Regarding the same excitation source (flash), its mounting on UAV and in the same axis as the infrared camera, would limit the experimental noise due to the impact of the inhomogeneity of illumination of the thermal scene.

Hence, even if the performances of such preliminary experiments need to be improved, it is interesting to note that active UAV-thermography can be implemented with simple averaging of large areas of interest or by considering a hot spot in order to estimate relative velocities with thermal methods. In the future, the progress related to the stability of the system will be obtained thanks to the new low-weight cameras more adapted to stationary flight.

\section{REFERENCES}

[1] Manohar A. F. L. "Determination of defect depth and size using virtual heat sources in impulse infrared thermography". Experimental Mechanics. 2012.

[2] Godin A. "Estimation sur des bases orthogonales des propriétés thermiques de matériaux hétérogènes à propriétés constantes par morceaux". Thèse de l'Université de Bordeaux I. 2012.

[3] Hay B. , Filz JR, Batsale JC. "Mesure de la diffusivité thermique par la méthode flash". Techniques de l'ingénieur, traité Mesures et Contrôle, r2955.

[4] Dolinko A. E. "Non-destructive visualization of defect borders in flawed plates inspected by thermal load". J. Phys. D: Appl. Phys.41. 2008.

[5] Grinzato E. V. V. "Quantitative infrared thermography in buildings". Energy and Buildings 29, 1-9. 1998.

[6] Mourand D., Batsale J.C., Non destructive evaluation with infrared cameras-two examples of real time estimation methods, Eurotherm 68-Poitiers -France,2001, 7 pages.

[7] Mourand D. "Contribution a la mise au point de methodes de controle non-destructif thermique. Traitement de signaux fortement bruites". Doctorate thesis Bordeaux 1998.

[8] Zarco-Tejadaa P.J., Diaz-Varelaa R., Angileria C., Loudjania V. P. "Tree height quantification using very high resolution imagery acquired from an unmanned aerial vehicle (UAV) and automatic 3D photo-reconstruction methods". European Journal of Agronomy 55, 89-99. 2014.

[9] Salvo G., Caruso L., Scordo A. "Urban Traffic Analysis through an UAV". Procedia-Social and Behavioral Sciences 111, 1083-1091. 2014.

[10] Guo F.S., Gao, W. "Batch Reconstruction from UAV Images with Prior Information". Acta Automatica Sinica 39(6), 834-845. 2013. 
[11] Michaelsen E., Meidow J. "Stochastic reasoning for structural pattern recognition: An example from imagebased UAV navigation". Pattern Recognition (In press:http://dx.doi.org/10.1016/j.patcog.2014.02.009). 2014.

[12] Siebert S., Teizer J. "Mobile 3D mapping for surveying earthwork projects using an Unmanned Aerial Vehicle (UAV) system". Automation in Construction 41, 1-14. 2014.

[13] Remondino F., Barazzetti L., Nex F., Scaioni M., Sarazzi D. "UAV photogrammetry for mapping and 3D modeling-current status and future perspectives" in edited proceedings by $\mathrm{H}$. Eisenbeiss, M. Kunz, H. Ingensand (Eds.), Proceedings of the International Conference on Unmanned Aerial Vehicle in Geomatics (UAV-g) 2011, Zurich, Switzerland (September 2011).

[14] Hudzietz B.P., Saripalli S. "An experimental evaluation of 3d terrain mapping with an autonomous helicopter". In edited proceedings by $\mathrm{H}$. Eisenbeiss, M. Kunz, H. Ingensand (Eds.), Proceedings of the International Conference on Unmanned Aerial Vehicle in Geomatics (UAV-g) 2011, Zurich, Switzerland (September 2011).

[15] Calderón R., Navas-Cortés J.A., Lucena C., Zarco-Tejada P.J. "High-resolution airborne hyperspectral and thermal imagery for early detection of Verticillium wilt of olive using fluorescence, temperature and narrow-band spectral indices". Remote Sensing of Environment 139, 231-245. 2013.

[16] Cassana J., Kantner J., Wiewel A., Cothren J "Archaeological aerial thermography: a case study at the Chacoera Blue J community, New Mexico". Journal of Archaelogical Science 45, 207-219. 2014.

[17] Philippi I., Batsale J.C., Maillet D., Degiovanni A., "Measurement of thermal diffusivity trough processing of infrared images" - Rev. Sci. Instrum. 66(1), pp182-192. 1995.

[18] Bamford M., Batsale J.C., Rungoat D., Fudym O., "Two dimensional velocity and diffusion mapping in the case of three-dimensional transient diffusion: Flash method and infrared image sequence analysis”, QIRT 2006. 